\title{
ON THE FORCE BETWEEN A DIELECTRIC CYLINDER IN A CONSTANT ELECTRIC FIELD AND A CONDUCTING HALF SPACE
}

\author{
$\mathrm{BY}$ \\ GUIDO DHONDT AND MANFRED KÖHL
}

Theoretical Research Associates, Enzianstraße 14, D-82194 Gröbenzell, Germany

\begin{abstract}
The force between an infinitely long dielectric cylinder in a constant electric field and a conducting half space is determined using the separation of variables technique on the Laplace equation in bipolar coordinates. The force is obtained as a series containing the relative distance between the cylinder and the half space as a parameter. This series is not uniformly convergent for the cylinder approaching the half space and the corresponding force cannot be obtained by performing the limit term per term. A special asymptotic analysis is presented leading to an analytic expression for this limiting value of the force.
\end{abstract}

1. Introduction. The change of the electric field due to the introduction of a conducting cylinder in a constant electric field bordering a conducting half space has been treated earlier [1]. In this paper a homogeneous isotropic dielectric cylinder is considered (see also [2]). While the determination of the electric field and resulting force between the cylinder and the half space is straightforward if using the appropriate coordinate system, the calculation of the limiting value of the force for the cylinder approaching the half space is more intricate. Indeed, the series solution representing the force is not uniformly convergent in this limit. In [2] it was shown by a numerical calculation that the limit cannot be taken term per term. This pure mathematical problem is solved by a very careful asymptotic analysis, which could be useful for other similar series as well.

2. Basic theory. The problem is sketched in Fig. 1. An infinitely long homogeneous isotropic dielectric cylinder with dielectric constant $\varepsilon$ is introduced in a half infinite space with dielectric constant $\varepsilon_{0}$ filled with a constant electric field $\vec{E}$. The remaining half space is a perfect conductor. The axis of the cylinder is parallel to the boundary between both half spaces. The distance between the center of the cylinder and the boundary is $h$. The resulting electric field and the force between the cylinder and the conducting half space are looked for.

Received September 7, 1994.

1991 Mathematics Subject Classification. Primary 78A30, 40G99, 40A30. 


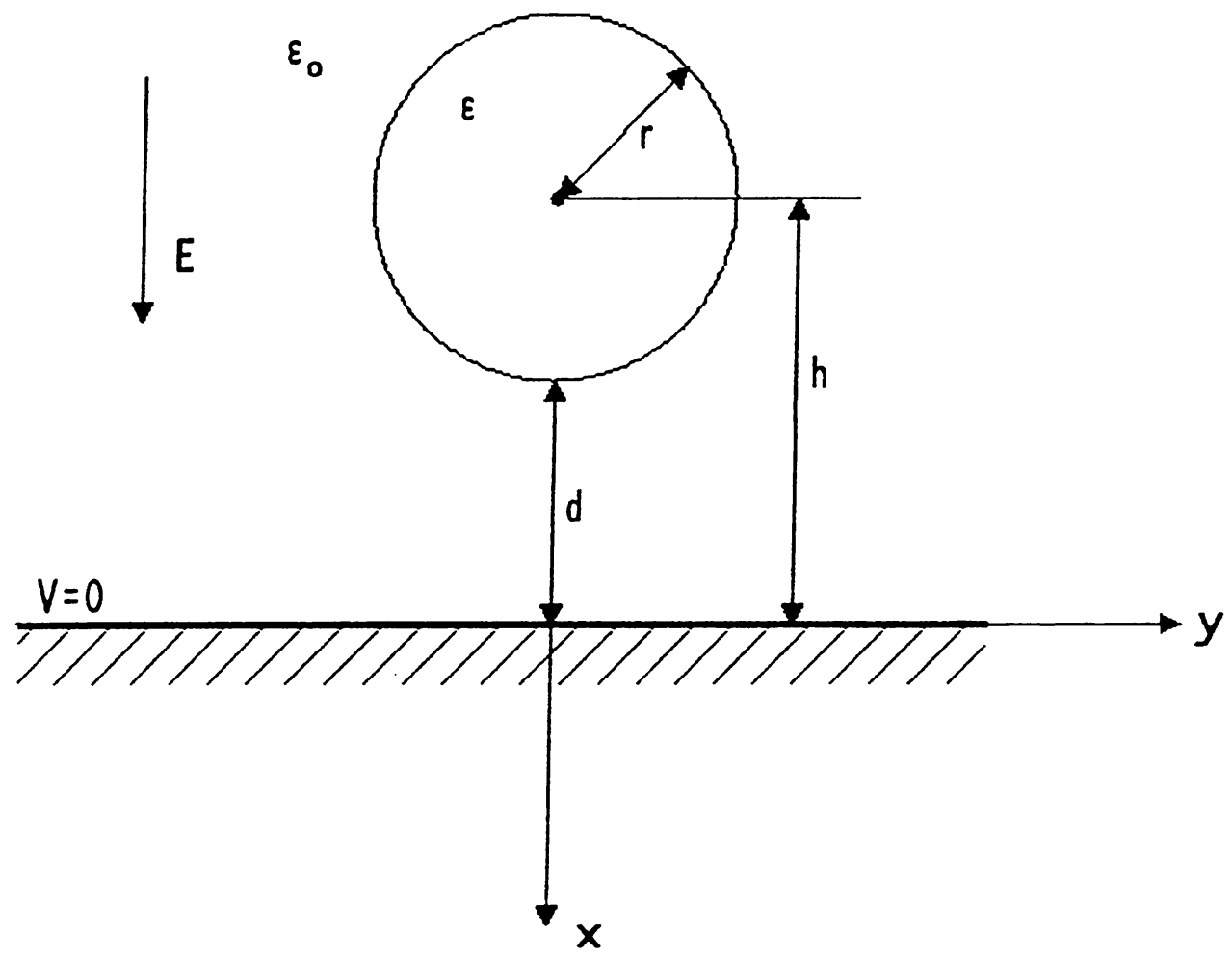

FIG. 1. Problem configuration

The problem is essentially two-dimensional. The potential field inside $\left(V_{i}\right)$ and outside $\left(V_{0}\right)$ the cylinder can be written as the sum of the potential field $V$ due to the constant electric field and a disturbance due to the introduction of the dielectric cylinder:

$$
\begin{aligned}
& V_{i}=V+V_{d i}, \\
& V_{o}=V+V_{d o}
\end{aligned}
$$

where $(\nabla$ is the nabla vector differential operator):

$$
\vec{E}=-\nabla V .
$$

Since the cylinder is homogeneous, isotropic and free from external charges, the basic equation inside and outside the cylinder is the Laplace equation [3]:

$$
\begin{aligned}
& \Delta V_{d i}=0, \\
& \Delta V_{d o}=0 .
\end{aligned}
$$

The boundary conditions amount to:

1. The field must be symmetric about the $x$-axis.

2. $V_{d i}$ must remain finite. 


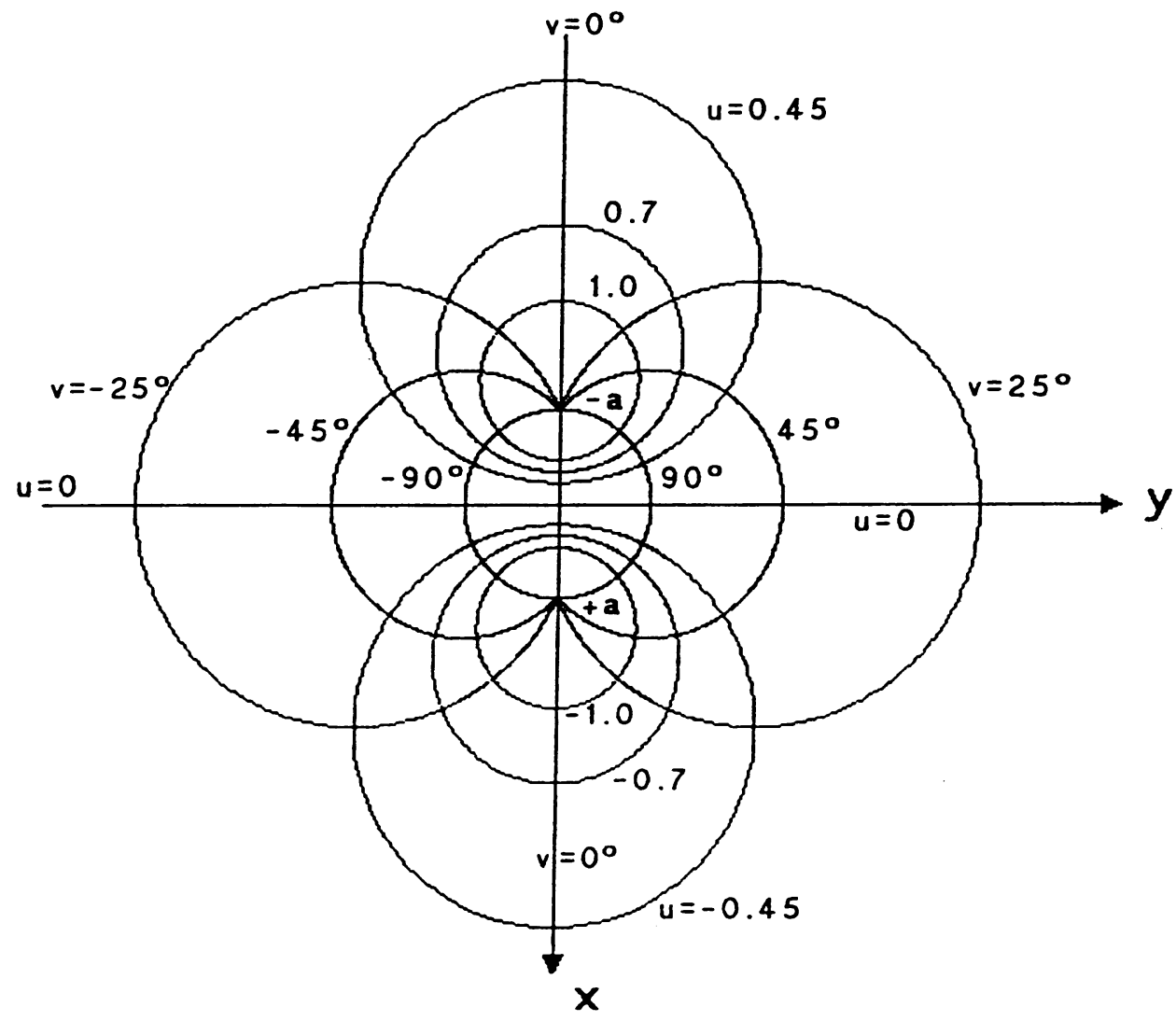

FIG. 2. Bipolar coordinate system

3. $V_{o}=0$ on the boundary between both half spaces.

4. The potential is continuous on the surface of the cylinder:

$$
V_{i}=V_{o}
$$

5. The normal component of the electric displacement is continuous on the surface of the cylinder:

$$
\varepsilon \frac{\partial V_{i}}{\partial n}=\varepsilon_{0} \frac{\partial V_{o}}{\partial n}
$$

For the present geometry the use of bipolar coordinates $(u, v)$ is very well suited (Fig. 2, [4]). The cartesian coordinates $(x, y)$ can be expressed as

$$
\begin{aligned}
& x(u, v)=-a \frac{\sinh u}{\cosh u-\cos v}, \\
& y(u, v)=a \frac{\sin v}{\cosh u-\cos v} .
\end{aligned}
$$


Taking the surface of the cylinder as $u=u_{0}, h$ and $r$ amount to (Figs. 1, 2):

$$
\begin{aligned}
& h=\frac{1}{2}\left[x\left(u_{0}, v=0\right)+x\left(u_{0}, v=\pi\right)\right]=\frac{a \cosh u_{0}}{\sinh u_{0}}, \\
& r=\frac{1}{2}\left[x\left(u_{0}, v=0\right)-x\left(u_{0}, v=\pi\right)\right]=\frac{a}{\sinh u_{0}}
\end{aligned}
$$

The metric tensor components are ([5]):

$$
\begin{gathered}
g_{u u}=g_{v v}=\frac{a^{2}}{(\cosh u-\cos v)^{2}}, \\
g_{u v}=g_{v u}=0,
\end{gathered}
$$

leaving the cartesian form of the Laplace equation unchanged, e.g., for $V_{d i}$ :

$$
\frac{\partial^{2} V_{d i}}{\partial u^{2}}+\frac{\partial^{2} V_{d i}}{\partial v^{2}}=0
$$

The electric field $\vec{E}_{d i}$ takes the form

$$
\vec{E}_{d i}=-\nabla V_{d i}=-\left\{\frac{1}{\sqrt{g_{u u}}} \frac{\partial V_{d i}}{\partial u} \vec{e}_{u}+\frac{1}{\sqrt{g_{v v}}} \frac{\partial V_{d i}}{\partial v} \vec{e}_{v}\right\}
$$

where

$$
\begin{aligned}
& \vec{e}_{u}=\frac{1}{\sqrt{g_{u u}}}\left\{\frac{\partial x}{\partial u} \vec{i}_{x}+\frac{\partial y}{\partial u} \vec{i}_{y}\right\}, \\
& \vec{e}_{v}=\frac{1}{\sqrt{g_{v v}}}\left\{\frac{\partial x}{\partial v} \vec{i}_{x}+\frac{\partial y}{\partial v} \vec{i}_{y}\right\} .
\end{aligned}
$$

$\vec{e}_{u}, \vec{e}_{v}$ are unit vectors in the bipolar coordinate system, $\vec{i}_{x}, \vec{i}_{y}$ in the cartesian system. Using separation of variables the solution of Eq. (14) can be written as

$$
V_{d i}=\sum_{n=0}^{\infty}\left(A_{n}^{\prime} e^{n u}+B_{n}^{\prime} e^{-n u}\right)\left(C_{n}^{\prime} \cos n v+D_{n}^{\prime} \sin n v\right)
$$

and similarly for $V_{d o}$. The symmetry about the $x$-axis and the finiteness of $V_{d i}$ lead to

$$
\begin{gathered}
V_{i}=E a \frac{\sinh u}{\cosh u-\cos v}+\sum_{n=0}^{\infty} C_{n} e^{-n u} \cos n v \\
V_{0}=E a \frac{\sinh u}{\cosh u-\cos v}+\sum_{n=0}^{\infty}\left(A_{n} e^{n u}+B_{n} e^{-n u}\right) \cos n v
\end{gathered}
$$

where the first term in each expression is due to the original constant electric field (expressed in bipolar coordinates). A zero potential on the boundary of both half spaces requires

$$
A_{n}=-B_{n}, \quad n=0,1,2, \ldots
$$


whereas Eq. (6) leads to

$$
C_{n}=A_{n} e^{2 n u_{0}}+B_{n}, \quad n=0,1,2, \ldots
$$

Equation (7) can be rewritten as

$$
\varepsilon \frac{\partial V_{i}}{\partial u}=\varepsilon_{0} \frac{\partial V_{o}}{\partial u}
$$

yielding

$$
C_{n}=\frac{\varepsilon_{0}}{\varepsilon}\left(B_{n}-A_{n} e^{2 n u_{0}}\right)+\left(\frac{\varepsilon_{0}}{\varepsilon}-1\right) 2 E a, \quad n=1,2,3, \ldots
$$

To get Eq. (24), $V$ has been rewritten as ([6], formula 1.461.2):

$$
V=E a \frac{\sinh u}{\cosh u-\cos v}=E a\left(1+2 \sum_{n=1}^{\infty} e^{-n u} \cos n v\right), \quad u>0
$$

The unknown constants can be solved from Eqs. (21), (22), and (24):

$$
\begin{aligned}
A_{n}=-B_{n} & =\frac{\left(\varepsilon_{0}-\varepsilon\right) 2 E a}{\varepsilon\left(e^{2 n u_{0}}-1\right)+\varepsilon_{0}\left(e^{2 n u_{0}}+1\right)}, \quad n=1,2,3, \ldots, \\
C_{n} & =\frac{\left(e^{2 n u_{0}}-1\right)\left(\varepsilon_{0}-\varepsilon\right) 2 E a}{\varepsilon\left(e^{2 n u_{0}}-1\right)+\varepsilon_{0}\left(e^{2 n u_{0}}+1\right)}, \quad n=1,2,3, \ldots, \\
C_{0} & =0,
\end{aligned}
$$

leading to

$$
\begin{gathered}
V_{i}=E a\left[1+4 \varepsilon_{0} \sum_{n=1}^{\infty} \frac{e^{-n u} \cos n v}{\varepsilon\left(1-e^{-2 n u_{0}}\right)+\varepsilon_{0}\left(1+e^{-2 n u_{0}}\right)}\right], \\
V_{0}=E a\left[1+2 \sum_{n=1}^{\infty} \frac{\left(\varepsilon_{0}-\varepsilon\right) e^{n u} e^{-2 n u_{0}}+\left(\varepsilon_{0}+\varepsilon\right) e^{-n u}}{\varepsilon\left(1-e^{-2 n u_{0}}\right)+\varepsilon_{0}\left(1+e^{-2 n u_{0}}\right)} \cos n v\right] .
\end{gathered}
$$

Taking the limit for $\varepsilon \rightarrow \infty$, and switching the limit with the summation sign (allowed due to the uniform convergence of the above series) leads to the expressions obtained by H. Jenss [1] for the conducting cylinder.

3. Force acting on the cylinder. The mechanical stress vector at some point of the cylinder amounts to [7]:

$$
\vec{t}=\frac{1}{2}\left(\varepsilon_{0}-\varepsilon\right)\left(\vec{E}_{i} \cdot \vec{E}_{0}\right) \vec{e}_{u}
$$


leading to the force

$$
\vec{K}=\frac{1}{2}\left(\varepsilon_{0}-\varepsilon\right) \int_{v=0}^{2 \pi}\left(\vec{E}_{i} \cdot \vec{E}_{0}\right) \vec{e}_{u} \sqrt{g_{v v}} d v
$$

for the complete cylinder, per unit length. Using Eq. (15), the cartesian components of the force yield

$$
\begin{aligned}
& K_{x}=\frac{1}{2 a}\left(\varepsilon_{0}-\varepsilon\right) \int_{0}^{2 \pi}\left[\frac{\varepsilon_{0}}{\varepsilon}\left(\left.\frac{\partial V_{o}}{\partial u}\right|_{u=u_{0}}\right)^{2}+\left(\left.\frac{\partial V_{o}}{\partial v}\right|_{u=u_{0}}\right)^{2}\right]\left(\cosh u_{0} \cos v-1\right) d v \\
& K_{y}=\frac{1}{2 a}\left(\varepsilon_{0}-\varepsilon\right) \int_{0}^{2 \pi}\left[\frac{\varepsilon_{0}}{\varepsilon}\left(\left.\frac{\partial V_{o}}{\partial u}\right|_{u=u_{0}}\right)^{2}+\left(\left.\frac{\partial V_{o}}{\partial v}\right|_{u=u_{0}}\right)^{2}\right]\left(-\sinh u_{0} \sin v\right) d v
\end{aligned}
$$

Finally, substitution of (27) and (28) into (31) and (32) leads to

$$
\begin{gathered}
K_{x}=2 \pi \varepsilon_{0}\left(\varepsilon_{0}^{2}-\varepsilon^{2}\right) E^{2} r \sinh u_{0} \\
\cdot \sum_{n=1}^{\infty} \frac{n(n+1) \cosh u_{0}\left(\varepsilon \sinh n u_{0}+\varepsilon_{0} \cosh n u_{0}\right)-n^{2}\left(\varepsilon \sinh (n+1) u_{0}+\varepsilon_{0} \cosh (n+1) u_{0}\right)}{\left(\varepsilon \sinh n u_{0}+\varepsilon_{0} \cosh n u_{0}\right)^{2}\left(\varepsilon \sinh (n+1) u_{0}+\varepsilon_{0} \cosh (n+1) u_{0}\right)} \\
K_{y}=0 .
\end{gathered}
$$

Equation (34) is a natural consequence of the symmetry about the $x$-axis. In Table 1 the dimensionless force $K_{x}^{*}$ defined by

$$
K_{x}^{*} \doteq \frac{K_{x}}{E^{2} \varepsilon_{0} r}
$$

has been calculated for several values of $u_{0}$ and $\varepsilon_{r} \doteq \varepsilon / \varepsilon_{0}$. Since

$$
d / r=\cosh u_{0}-1
$$

$u_{0}$ is a measure of $d$ for constant $r$, i.e., $d \rightarrow 0$ for $u_{0} \rightarrow 0$ and $r$ constant. Table 1 shows that for finite $\varepsilon_{r}$ and $u_{0} \rightarrow 0$ (i.e., the cylinder approaches the conducting half space) the force remains finite, whereas for $\varepsilon_{r} \rightarrow \infty, u_{0} \rightarrow 0$ the values of $K_{x}^{*}$ seem to blow up like $\pi / u_{0}$. The latter case corresponds to a conducting cylinder approaching the half space and will be considered first since it is the simpler case. 
TABLE 1. Numerical values for the dimensionless force (the symbol "-" signifies that no value was calculated).

\begin{tabular}{|r|r|r|r|r|r|}
\hline$u_{0}$ & $d / r$ & $K_{x}^{*}\left(\varepsilon_{r}=5\right)$ & $K_{x}^{*}\left(\varepsilon_{r}=10\right)$ & $K_{x}^{*}\left(\varepsilon_{r}=100\right)$ & $K_{x}^{*} u_{0} / \pi\left(\varepsilon_{r} \rightarrow \infty\right)$ \\
\hline 10.0 & $1.110^{4}$ & $5.3010^{-13}$ & $7.9610^{-13}$ & $1.1410^{-12}$ & $3.7910^{-12}$ \\
\hline 5.0 & 73.21 & $1.7110^{-6}$ & $2.5710^{-6}$ & $3.6910^{-6}$ & $6.1110^{-6}$ \\
\hline 2.0 & 2.762 & $1.3410^{-2}$ & $2.0410^{-2}$ & $2.9410^{-2}$ & 0.0195 \\
\hline 1.5 & 1.352 & $5.7510^{-2}$ & $8.8110^{-2}$ & 0.1287 & 0.0641 \\
\hline 1.0 & 0.543 & 0.2331 & 0.3688 & 0.5587 & 0.1863 \\
\hline 0.5 & 0.128 & 0.8807 & 1.5650 & 2.7260 & 0.4630 \\
\hline 0.25 & $3.1410^{-2}$ & 1.6507 & 3.4730 & - & 0.8651 \\
\hline 0.10 & $5.0010^{-3}$ & 2.2444 & 5.7813 & 21.916 & 0.9305 \\
\hline 0.05 & $1.2510^{-3}$ & - & 6.6587 & - & 0.9441 \\
\hline 0.04 & $8.0010^{-4}$ & - & - & - & 0.9579 \\
\hline 0.03 & $4.5010^{-4}$ & - & - & 56.149 & 0.9858 \\
\hline 0.01 & $5.0010^{-5}$ & 2.4413 & 7.0757 & 86.784 & - \\
\hline 0.005 & $1.2510^{-5}$ & - & - & 95.915 & 0.9986 \\
\hline 0.001 & $5.0010^{-7}$ & 2.44357 & 7.0956 & 100.226 & - \\
\hline 0.0005 & $1.2510^{-7}$ & - & 7.09574 & 100.381 & 0.9998 \\
\hline 0.0001 & $5.0010^{-9}$ & 2.44360 & 7.09578 & 100.431 & - \\
\hline
\end{tabular}

\section{Limit value of the force for a conducting cylinder approaching the half} space. In this section the asymptotic behaviour of $K_{x}^{*}$ for $\varepsilon_{r} \rightarrow \infty$ and $u_{0} \rightarrow 0$ is looked at. For the conducting cylinder the nondimensional form of Eq. (33) yields

$$
K_{x}^{*}=-2 \pi \sinh u_{0} \sum_{n=1}^{\infty} \frac{n(n+1) \cosh u_{0} \sinh n u_{0}-n^{2} \sinh (n+1) u_{0}}{\sinh ^{2} n u_{0} \sinh (n+1) u_{0}}
$$

The statement at the end of the last section amounts to

$$
\lim _{u_{0} \rightarrow 0} \frac{-K_{x}^{*}}{2 \pi} u_{0}=-\frac{1}{2}
$$

which has to be proven. The main problem in taking the limit of Eq. (37) for $u_{0} \rightarrow 0$ is that the limit and summation sign cannot be switched. Indeed, it is not difficult to check that the limit for each single term in the series for $u_{0} \rightarrow 0$ tends to zero (for $n$ fixed). This phenomenon can be better understood if one realizes that the series is not uniformly convergent in $u_{0}$ over the interval $[0, \alpha]$ where $\alpha$ is some positive real number. No matter how small $u_{0}$ is chosen it is always possible to find a value of $n$ big enough to yield a significant contribution of $n u_{0}$. The key in the present asymptotic approach is that, although $u_{0} \rightarrow 0$, no assumption is made about the size of $n u_{0}$. Looking at the expression

$$
\begin{aligned}
I \doteq \frac{-K_{x}^{*}}{2 \pi} u_{0} & =u_{0} \sinh u_{0} \sum_{n=1}^{\infty} \frac{n(n+1) \cosh u_{0} \sinh n u_{0}-n^{2} \sinh (n+1) u_{0}}{\sinh ^{2} n u_{0} \sinh (n+1) u_{0}} \\
& \doteq u_{0} \sinh u_{0} \sum_{n=1}^{\infty} \frac{N_{n}}{D_{n}}
\end{aligned}
$$


one can write

$$
\begin{aligned}
& N_{n}=n \cosh u_{0} \sinh n u_{0}-n^{2} \cosh n u_{0} \sinh u_{0} \\
& \sim n \sinh n u_{0}-n^{2} u_{0} \cosh n u_{0}, \quad u_{0} \rightarrow 0 \\
& \sim n \sum_{k=0}^{\infty}\left(n u_{0}\right)^{2 k+1}\left\{\frac{1}{(2 k+1) !}-\frac{1}{(2 k) !}\right\}, \quad u_{0} \rightarrow 0 \\
& \sim-n \sum_{k=1}^{\infty}\left(n u_{0}\right)^{2 k+1} \frac{2 k}{(2 k+1) !}, \quad u_{0} \rightarrow 0, \\
& D_{n} \sim \sinh ^{2} n u_{0}\left[e^{n u_{0}}\left(1+u_{0}\right)-e^{-n u_{0}}\left(1-u_{0}\right)\right] / 2, \quad u_{0} \rightarrow 0 \\
& \sim \sinh ^{3} n u_{0}, \quad u_{0} \rightarrow 0 .
\end{aligned}
$$

Hence

$$
I \sim-u_{0}^{2} \sum_{k=1}^{\infty} u_{0}^{2 k+1} \frac{2 k}{(2 k+1) !} \sum_{n=1}^{\infty} \frac{n^{2 k+2}}{\sinh ^{3} n u_{0}}, \quad u_{0} \rightarrow 0 .
$$

Since according to [8]

$$
\begin{aligned}
\frac{1}{\sinh ^{3} n u_{0}} & =\frac{8}{e^{3 n u_{0}}\left(1-e^{-2 n u_{0}}\right)^{3}} \\
& =8 \sum_{i=0}^{\infty} \frac{(i+1)(i+2)}{2} e^{-(2 i+3) n u_{0}}
\end{aligned}
$$

Eq. (42) is equivalent to

$$
I \sim-8 u_{0}^{2} \sum_{k=1}^{\infty} u_{0}^{2 k+1} \frac{2 k}{(2 k+1) !} \sum_{i=0}^{\infty} \frac{(i+1)(i+2)}{2} \sum_{n=1}^{\infty} n^{2 k+2}\left(e^{-(2 i+3) u_{0}}\right)^{n}, \quad u_{0} \rightarrow 0 .
$$

Defining

$$
S_{k} \doteq \sum_{n=1}^{\infty} n^{k} q^{n}
$$

it is not difficult to prove that

$$
S_{k}=q \frac{d S_{k-1}}{d q}
$$

Using this expression and

$$
S_{0}=\frac{q}{1-q},
$$

it is possible to find an explicit expression for $S_{k}$ :

$$
S_{k}=\frac{\sum_{i=1}^{k} a_{i}^{(k)} q^{i}}{(1-q)^{k+1}}
$$

where the coefficients $a_{i}^{(k)}$ are determined by a recursive relation:

$$
\begin{aligned}
& a_{1}^{(k)}=a_{k}^{(k)}=1 \\
& a_{j}^{(k)}=j a_{j}^{(k-1)}+(k+1-j) a_{j-1}^{(k-1)}, \quad j=2, \ldots, k-1 .
\end{aligned}
$$


Equation (49) also yields

$$
\sum_{i=1}^{k} a_{i}^{(k)}=k \sum_{i=1}^{k-1} a_{i}^{(k-1)}
$$

leading to

$$
\sum_{i=1}^{k} a_{i}^{(k)}=k !
$$

Using Eq. (48) in Eq. (44) leads to

$$
I \sim-8 u_{0}^{2} \sum_{k=1}^{\infty} u_{0}^{2 k+1} \frac{2 k}{(2 k+1) !} \sum_{i=0}^{\infty} \frac{(i+1)(i+2)}{2} \frac{\sum_{j=1}^{2 k+2} a_{j}^{(2 k+2)}\left[e^{-(2 i+3) u_{0}}\right]^{j}}{\left[(2 i+3) u_{0}\right]^{2 k+3}}, \quad u_{0} \rightarrow 0
$$

or, using Eq. (51),

$$
I \sim-8 \sum_{i=0}^{\infty} \frac{(i+1)(i+2)}{(2 i+3)^{3}} \sum_{k=1}^{\infty} \frac{k(2 k+2)}{\left[(2 i+3)^{2}\right]^{k}}, \quad u_{0} \rightarrow 0 .
$$

The expression in $k$ is again of the form (45). Summing explicitly yields

$$
I \sim-\frac{1}{2} \sum_{i=0}^{\infty} \frac{2 i+3}{(i+1)^{2}(i+2)^{2}}, \quad u_{0} \rightarrow 0 .
$$

After splitting into partial fractions the calculation is complete:

$$
\begin{aligned}
I & \sim-\frac{1}{2} \sum_{i=0}^{\infty}\left\{\frac{1}{(i+1)^{2}}-\frac{1}{(i+2)^{2}}\right\}, \quad u_{0} \rightarrow 0 \\
& \sim-\frac{1}{2}, \quad u_{0} \rightarrow 0 .
\end{aligned}
$$

It demonstrates that the first term in the asymptotic expansion of $K_{x}^{*}$ (Eq. (37)) in $u_{0}$ is indeed $\pi / u_{0}$.

5. Limit value of the force for a dielectric cylinder. For a dielectric cylinder, Eq. (33) can be written in the form (using $\varepsilon_{r} \doteq \varepsilon / \varepsilon_{0}$ and (35)):

$$
\begin{aligned}
K_{x}^{*}= & 2 \pi\left(1-\varepsilon_{r}^{2}\right) \sinh u_{0} \\
& \cdot \sum_{n=1}^{\infty} \frac{\left[n(n+1) \cosh u_{0}\left(\varepsilon_{r} \sinh n u_{0}+\cosh n u_{0}\right)-n^{2}\left(\varepsilon_{r} \sinh (n+1) u_{0}+\cosh (n+1) u_{0}\right)\right]}{\left(\varepsilon_{r} \sinh n u_{0}+\cosh n u_{0}\right)^{2}\left[\varepsilon_{r} \sinh (n+1) u_{0}+\cosh (n+1) u_{0}\right]} \\
\doteq & 2 \pi\left(1-\varepsilon_{r}^{2}\right) \sinh u_{0} \sum_{n=1}^{\infty} \frac{N_{n}}{D_{n}} .
\end{aligned}
$$

$N_{n}$ and $D_{n}$ now take the form

$N_{n} \sim \varepsilon_{r} n\left[\sinh n u_{0}-\left(n u_{0}\right) \cosh n u_{0}\right]+n\left[\cosh n u_{0}-\left(n u_{0}\right) \sinh n u_{0}\right]+O\left(u_{0}^{2}\right), \quad u_{0} \rightarrow 0$

$$
D_{n} \sim\left(\varepsilon_{r} \sinh n u_{0}+\cosh n u_{0}\right)^{3}\left[1+u_{0} \frac{\varepsilon_{r} \cosh n u_{0}+\sinh n u_{0}}{\varepsilon_{r} \sinh n u_{0}+\cosh n u_{0}}\right], \quad u_{0} \rightarrow 0 .
$$


Consequently the leading term of $K_{x}^{*} /\left[2 \pi\left(1-\varepsilon_{r}^{2}\right)\right], u_{0} \rightarrow 0$, yields

$$
J_{-1} \doteq \sinh u_{0} \sum_{n=1}^{\infty} \frac{\varepsilon_{r} n\left[\sinh n u_{0}-\left(n u_{0}\right) \cosh n u_{0}\right]+n\left[\cosh n u_{0}-\left(n u_{0}\right) \sinh n u_{0}\right]}{\left[\varepsilon_{r} \sinh n u_{0}+\cosh n u_{0}\right]^{3}}
$$

The numerator of a typical term in Eq. (59) can be written as

$$
\sinh u_{0}\left\{-\varepsilon_{r} n \sum_{k=0}^{\infty}\left(n u_{0}\right)^{2 k+1} \frac{2 k}{(2 k+1) !}-n \sum_{k=0}^{\infty}\left(n u_{0}\right)^{2 k} \frac{2 k-1}{(2 k) !}\right\}
$$

and the denominator yields

$$
\frac{1}{\left(\varepsilon_{r} \sinh n u_{0}+\cosh n u_{0}\right)^{3}}=\frac{8}{\left(\varepsilon_{r}+1\right)^{3}} \sum_{i=0}^{\infty} \frac{(i+1)(i+2)}{2}\left(\frac{\varepsilon_{r}-1}{\varepsilon_{r}+1}\right)^{i} e^{-(2 i+3) n u_{0}}
$$

using the same strategy as for the conducting cylinder, $J_{-1}$ is transformed into (comparable to Eq. (54))

$$
\begin{aligned}
J_{-1} \sim \frac{1}{u_{0}}\{- & \frac{\varepsilon_{r}}{2\left(\varepsilon_{r}+1\right)^{3}} \sum_{i=0}^{\infty}\left(\frac{\varepsilon_{r}-1}{\varepsilon_{r}+1}\right)^{i}\left[\frac{1}{(i+1)^{2}}-\frac{1}{(i+2)^{2}}\right] \\
& -\frac{1}{2\left(\varepsilon_{r}+1\right)^{3}} \sum_{i=0}^{\infty}\left(\frac{\varepsilon_{r}-1}{\varepsilon_{r}+1}\right)^{i}\left[\frac{1}{(i+1)^{2}}+\frac{1}{(i+2)^{2}}\right] \\
& \left.+\frac{1}{\left(\varepsilon_{r}+1\right)^{3}} \sum_{i=0}^{\infty}\left(\frac{\varepsilon_{r}-1}{\varepsilon_{r}+1}\right)^{i}\right\}, \quad u_{0} \rightarrow 0,
\end{aligned}
$$

which vanishes for a finite $\varepsilon_{r}$ (can be checked through appropriate combination of terms). This shows that the leading term of $K_{x}^{*}$ remains finite. The next term of $K_{x}^{*} /\left[2 \pi\left(1-\varepsilon_{r}^{2}\right)\right]$ yields

$$
J_{0} \doteq-u_{0} \sinh u_{0} \sum_{n=1}^{\infty} \frac{N_{n}\left[\varepsilon_{r} \cosh n u_{0}+\sinh n u_{0}\right]}{\left[\varepsilon_{r} \sinh n u_{0}+\cosh n u_{0}\right]^{4}}
$$

where $N_{n}$ has the form (57). The numerator after the summation sign amounts to

$$
\begin{aligned}
& N_{n}\left[\varepsilon_{r} \cosh n u_{0}+\sinh n u_{0}\right]=n\left\{\frac{\varepsilon_{r}^{2}+1}{2}-\varepsilon_{r}\left(n u_{0}\right)\right\} \sinh \left(2 n u_{0}\right) \\
& +n\left\{\varepsilon_{r}-\left(n u_{0}\right)\left(\frac{\varepsilon_{r}^{2}+1}{2}\right)\right\} \cosh \left(2 n u_{0}\right)+n\left(n u_{0}\right)\left(\frac{1-\varepsilon_{r}^{2}}{2}\right) .
\end{aligned}
$$

Substitution of Eq. (64) into Eq. (63) yields

$$
\begin{aligned}
J_{0}=- & u_{0} \sinh u_{0}\left\{\left(\frac{\varepsilon_{r}^{2}+1}{2}\right) L(0,1)-\varepsilon_{r} L(1,1)\right. \\
& \left.+\varepsilon_{r} L(0,0)-\left(\frac{\varepsilon_{r}^{2}+1}{2}\right) L(1,0)+\left(\frac{1-\varepsilon_{r}^{2}}{2}\right) \sum_{n=1}^{\infty} \frac{n\left(n u_{0}\right)}{\left[\varepsilon_{r} \sinh n u_{0}+\cosh n u_{0}\right]^{4}}\right\}
\end{aligned}
$$


where

$$
L(\beta, \gamma) \doteq \sum_{n=1}^{\infty} n\left(n u_{0}\right)^{\beta} \frac{1}{\left(\varepsilon_{r} \sinh n u_{0}+\cosh n u_{0}\right)^{4}} \sum_{k=0}^{\infty} \frac{\left(2 n u_{0}\right)^{2 k+\gamma}}{(2 k+\gamma) !}
$$

Analogously as for the conducting cylinder one can show that

$$
\begin{aligned}
& L(\beta, \gamma) \sim \frac{4}{u_{0}^{2}\left(\varepsilon_{r}+1\right)^{4}} \\
& \quad \cdot \sum_{i=0}^{\infty} \frac{(i+1)(i+3)}{6(i+2)}\left\{\frac{1}{2^{\beta}(i+2)^{\beta+\gamma}} \sum_{k=0}^{\infty} \frac{\prod_{j=1}^{\beta+1}(2 k+\gamma+j)}{\left[(i+2)^{2}\right]^{k}}\right\}\left(\frac{\varepsilon_{r}-1}{\varepsilon_{r}+1}\right)^{i}, \quad u_{0} \rightarrow 0
\end{aligned}
$$

and

$$
\begin{array}{r}
\sum_{n=1}^{\infty} \frac{n\left(n u_{0}\right)}{\left(\varepsilon_{r} \sinh n u_{0}+\cosh n u_{0}\right)^{4}} \sim \frac{4}{u_{0}^{2}\left(\varepsilon_{r}+1\right)^{4}} \sum_{i=0}^{\infty} \frac{(i+1)(i+3)}{6(i+2)}\left(\frac{\varepsilon_{r}-1}{\varepsilon_{r}+1}\right)^{i} \frac{1}{i+2} \\
u_{0} \rightarrow 0
\end{array}
$$

leading to the following expression for $J_{0}$ :

$$
J_{0} \sim-\frac{4}{\left(\varepsilon_{r}+1\right)^{4}} \sum_{i=0}^{\infty} \frac{(i+1)(i+3)}{6(i+2)}\left(\frac{\varepsilon_{r}-1}{\varepsilon_{r}+1}\right)^{i} M, \quad u_{0} \rightarrow 0
$$

where

$$
\begin{aligned}
M= & \left(\frac{\varepsilon_{r}^{2}+1}{2}\right) \frac{1}{i+2} \sum_{k=0}^{\infty} \frac{2 k+2}{(i+2)^{2 k}}-\frac{\varepsilon_{r}}{2(i+2)^{2}} \sum_{k=0}^{\infty} \frac{(2 k+3)(2 k+2)}{(i+2)^{2 k}} \\
& +\varepsilon_{r} \sum_{k=0}^{\infty} \frac{2 k+1}{(i+2)^{2 k}}-\left(\frac{\varepsilon_{r}^{2}+1}{2}\right) \frac{1}{2(i+2)} \sum_{k=0}^{\infty} \frac{(2 k+2)(2 k+1)}{(i+2)^{2 k}}+\frac{1}{i+2}\left(\frac{1-\varepsilon_{r}^{2}}{2}\right) .
\end{aligned}
$$

Through summation $M$ yields

$$
\begin{gathered}
M=\left(\frac{\varepsilon_{r}^{2}+1}{2}\right) \frac{1}{j}\left(\frac{2 j^{4}}{\left(j^{2}-1\right)^{2}}\right)-\varepsilon_{r} \frac{j^{2}\left(3 j^{2}+1\right)}{\left(j^{2}-1\right)^{3}}+\varepsilon_{r} \frac{j^{2}\left(j^{2}+1\right)}{\left(j^{2}-1\right)^{2}} \\
-\left(\frac{\varepsilon_{r}^{2}+1}{2}\right) \frac{1}{j}\left(\frac{j^{4}\left(j^{2}+3\right)}{\left(j^{2}-1\right)^{3}}\right)+\left(\frac{1-\varepsilon_{r}^{2}}{2}\right) \frac{1}{j}
\end{gathered}
$$

where $j=i+2$. Consequently,

$$
\begin{aligned}
J_{0} \sim- & \frac{4}{\left(\varepsilon_{r}+1\right)^{4}} \sum_{j=2}^{\infty} \frac{1}{6}\left(\frac{\varepsilon_{r}-1}{\varepsilon_{r}+1}\right)^{j-2}\left\{\left(\frac{\varepsilon_{r}^{2}+1}{2}\right) \frac{2 j^{2}}{\left(j^{2}-1\right)}-\varepsilon_{r} \frac{j\left(3 j^{2}+1\right)}{\left(j^{2}-1\right)^{2}}\right. \\
& \left.+\varepsilon_{r} \frac{j\left(j^{2}+1\right)}{\left(j^{2}-1\right)}-\left(\frac{\varepsilon_{r}^{2}+1}{2}\right) \frac{j^{2}\left(j^{2}+3\right)}{\left(j^{2}-1\right)^{2}}+\left(\frac{1-\varepsilon_{r}^{2}}{2}\right) \frac{j^{2}-1}{j^{2}}\right\}, \quad u_{0} \rightarrow 0 .
\end{aligned}
$$


Splitting into partial fractions yields:

$$
\begin{gathered}
\frac{2 j^{2}}{j^{2}-1}=2+\frac{1}{j-1}-\frac{1}{j+1}, \\
\frac{j\left(3 j^{2}+1\right)}{\left(j^{2}-1\right)^{2}}=\frac{-1+3 j}{2(j-1)^{2}}+\frac{1+3 j}{2(j+1)^{2}}, \\
\frac{j\left(j^{2}+1\right)}{\left(j^{2}-1\right)}=j+\frac{1}{j-1}+\frac{1}{j+1}, \\
\frac{j^{2}\left(j^{2}+3\right)}{\left(j^{2}-1\right)^{2}}=1+\frac{-1+3 j}{2(j-1)^{2}}-\frac{1+3 j}{2(j+1)^{2}} .
\end{gathered}
$$

Substitution of Eqs. (73)-(76) into Eq. (72) reveals that

$$
\begin{aligned}
& \sum_{j=2}^{\infty}\left(\frac{\varepsilon_{r}-1}{\varepsilon_{r}+1}\right)^{j-2}\left\{\left(\frac{\varepsilon_{r}^{2}+1}{2}\right) \frac{2 j^{2}}{\left(j^{2}-1\right)}-\varepsilon_{r} \frac{j\left(3 j^{2}+1\right)}{\left(j^{2}-1\right)^{2}}\right. \\
& \left.\quad+\varepsilon_{r} \frac{j\left(j^{2}+1\right)}{\left(j^{2}-1\right)}-\left(\frac{\varepsilon_{r}^{2}+1}{2}\right) \frac{j^{2}\left(j^{2}+3\right)}{\left(j^{2}-1\right)^{2}}\right\}=-2 \sum_{j=2}^{\infty}\left(\frac{\varepsilon_{r}-1}{\varepsilon_{r}+1}\right)^{j-2}\left(\frac{1-\varepsilon_{r}^{2}}{2}\right)\left(\frac{j^{2}-1}{j^{2}}\right)
\end{aligned}
$$

and, consequently,

$$
\begin{aligned}
J_{0} & \sim \frac{1-\varepsilon_{r}^{2}}{3\left(\varepsilon_{r}+1\right)^{4}} \sum_{j=2}^{\infty}\left(\frac{\varepsilon_{r}-1}{\varepsilon_{r}+1}\right)^{j-2}\left(1-\frac{1}{j^{2}}\right), \quad u_{0} \rightarrow 0, \\
& \sim \frac{1-\varepsilon_{r}^{2}}{3\left(\varepsilon_{r}+1\right)^{4}}\left\{\frac{\varepsilon_{r}+1}{2}-\sum_{j=2}^{\infty} \frac{1}{j^{2}}\left(\frac{\varepsilon_{r}-1}{\varepsilon_{r}+1}\right)^{j-2}\right\}, \quad u_{0} \rightarrow 0 .
\end{aligned}
$$

The series in Eq. (79) is related to $S_{-2}$ which can be obtained from $S_{0}$ through repeated application of the inverse operation of Eq. (46):

$$
S_{k-1}=\int_{0}^{q} \frac{S_{k}}{\xi} d \xi
$$

This yields, starting from $S_{0}$ in Eq. (47):

$$
\begin{aligned}
& S_{-1}=-\ln (1-q), \\
& S_{-2}=-\int_{0}^{q} \frac{\ln (1-\xi)}{\xi} d \xi,
\end{aligned}
$$

which cannot be expressed in elementary functions ([6], formula 2.728.2). So the final expression for $K_{x}^{*}$ is

$$
K_{x}^{*} \sim \frac{2 \pi}{3} \frac{\left(\varepsilon_{r}^{2}-1\right)^{2}}{\left(\varepsilon_{r}+1\right)^{4}}\left\{\frac{\varepsilon_{r}+1}{2}-\sum_{j=0}^{\infty} \frac{1}{(j+2)^{2}}\left(\frac{\varepsilon_{r}-1}{\varepsilon_{r}+1}\right)^{j}\right\}, \quad u_{0} \rightarrow 0 .
$$

Evaluation of Eq. (83) agrees with the values in Table 1 for $u_{0} \ll 1$. 
6. Conclusions. The force between a dielectric cylinder in a constant electric field and a conducting half space has been determined using the separation of variables technique on the Laplace equation in bipolar coordinates. The force has been obtained as a series containing the relative distance between the cylinder and the half space as a parameter. Due to the nonuniform convergence of the series in this parameter, a special asymptotic analysis was needed to find the limiting value of the force as the cylinder approaches the half space. It was shown that for a dielectric cylinder the force remains finite whereas for a conducting cylinder the force is inversely proportional to the relative distance.

\section{REFERENCES}

[1] H. Jenss, Das Potential isolierter Sonden im homogenen Felde, Archiv Elektrotechnik 26, 557-561 (1932)

[2] M. Köhl, Dielektrischer Zylinder im homogenen Feld bei Anwesenheit eines leitenden Halbraumes, Studienarbeit at the Institut für Theoretische Elektrotechnik, Technische Universität Berlin, unpublished, 1975

[3] J. D. Jackson, Classical Electrodynamics, John Wiley and Sons, 1975

[4] N. N. Lebedev, I. P. Skalskaya, and Y. S. Uflyand, Problems in Mathematical Physics, Pergamon Press, 1966

[5] A. Cemal Eringen, Mechanics of Continua, R. E. Krieger Publishing Company, 1980

[6] I. S. Gradshteyn and I. M. Ryzhik, Table of Integrals, Series and Products, Academic Press, 1980

[7] K. Simonyi, Theoretische Elektrotechnik, Deutscher Verlag der Wissenschaften, Berlin, 1973

[8] M. Abramowitz and I. A. Stegun, Handbook of Mathematical Functions, 1972 